\title{
Beam-colored Sketch and Image-based 3D Continuous Wireframe Reconstruction with different Materials and Cross-Sections
}

Martin Denk, Klemens Rother, Kristin Paetzold

The automated reverse engineering of wireframes is a common task in topology optimization, fast concept design, bionic and point cloud reconstruction. This article deals with the usage of skeleton-based reconstruction of sketches in 2D images. The result leads to a flexible at least $C_{1}$ continuous shape description.

Keywords: Reverse Engineering, Fast Concept Design, Skeleton

\section{Introduction}

Modern design projects with complex problems are often decomposed, and each part is tackled by a multidisciplinary design team (MDT) (Ensici and Badke-Schaub, 2011). Splitting the development into different parts is the simplest decision but comes with a high risk of a suboptimal solution (Song, 2004). In particular, in the early stage of product development, fast concept design is required, which should ensure a huge amount of conceptual design suggestions. With a huge number of simple sketches, the rough shape of the product is often estimated but not evaluated. Particularly, in modern lightweight designs, shapes derived from scanned point clouds (Berger et al., 2017) or from surfaces in finite element analysis (Ben Makhlouf et al., 2019; Louhichi et al., 2015) are necessary to redesign. Such shape is often computed numerically using structural optimization such as topology optimization (Changizi and Warn, 2020; Denk et al., 2020a), shape optimization (Bandara et al., 2016), or in some cases, multi-material optimization (Gao et al., 2020). For the reconstruction, a huge variety of different shape descriptor (Agathos et al., 2007; Guo et al., 2016) can be chosen, such as constructive solid geometry (CSG) (Bénière et al., 2013; Denk et al., 2019; Vidal et al., 2014) or freeform surfaces representing the boundary (Ben Makhlouf et al., 2019; Louhichi et al., 2015). In particular organic shapes resulting from topology optimization, a descriptor such as medial axes (skeletons) (Blum, 1967) shows desirable concepts for recent reverse engineering approaches in topology optimization (Mayer and Wartzack, 2020; Nana et al., 2017a; Stangl and Wartzack, 2015; Yin et al., 2020). These skeleton- 
based methods are capable of reasonable part segmentation (Agathos et al., 2007; Feng et al., 2015; Reniers and Telea, 2008), so that a hand can be segmented into five fingers and the palm of the hand (Wu et al., 2016). Such parametrization has been implemented in various applications for the reverse engineering task in topology optimization (Mayer and Wartzack, 2020; Nana et al., 2017a; Stangl and Wartzack, 2015; Yin et al., 2020) for predefined optimized shapes. Performing topology optimization requires knowledge in finite element analysis, mechanical engineering and access to software packages. Additionally, these results often need to be modified to fulfill geometric limitations for the manufacturing process such as 3D printing (Adam and Zimmer, 2015). The approach proposed in this article deals with a fast concept design using images that can result from topology optimization, hand-drawn sketches, or computer-aided design applications. If proof of strength or further shape optimization should be done, such shapes can directly be evaluated with a small amount of knowledge in mechanical engineering.

\section{State of the Art}

State of the Art is referred to skeleton-based reverse engineering and sketch-based reverse engineering strategies. Sketch-based reverse engineering deals with the 3D shape reconstruction using 2D sketches (Zhao et al., 2017). In the recent publications, the beamline of topology optimized shapes is approximated by a contraction method applied on a polygon mesh (Nana et al., 2017b; Stangl and Wartzack, 2015), a medial axis transformation (Mayer and Wartzack, 2020), or morphological thinning applied on images (Bremicker et al., 1991; Denk et al., 2020b; Yin et al., 2020). While these publications cover only the geometric shape, our approach also deals with different materials, predefined cross-sections, and, similar to (Denk et al., 2020b), a fully automated robust subdivision surface representation. Additionally, the use-cases are extended to also user-defined shapes for 2D images. Furthermore, we use similar to (Zhao et al., 2017) skeleton-based sketches for the reconstruction, which is extended using colored regions in the image similar to (Xu et al., 2013). Our work is mostly inspired by our recent work (Denk et al., 2020b). In that approach, shapes, as obtained by topology optimization, are reconstructed to a subdivision surface control grid by using the Euclidian distance transformation for circular control grids. In contrast, this work addresses the usage of different materials and automatic beam evaluation with a finite element method and also considering elliptical cross-sections. The following figure shows the fast concept design using colored cross-sections and the line thickness of a skeleton and its desirable 3D parametrization. 


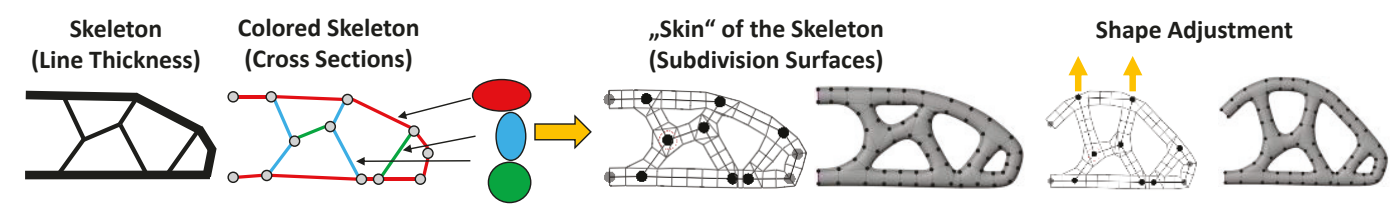

Figure 1: Coloured Shape Determination

\section{Sketch-based reconstruction}

As input, the user has to draw a skeleton-based sketch with optional colored lines and corresponding colored cross-sections. Based on the cross-section bounding box, we estimate the shape using subdivision surfaces and evaluate the suggested concept automatically using FEM. These subdivision surfaces are bounded by the skeleton so that adjusting the position of the skeleton knots also changes the resulting surface. To generate a wire frame representation, these images need to be transferred into wireframes enriched by the corresponding cross-sections. First, the extraction of the skeleton similar to (Denk et al., 2020b) is covered In the following chapters. The image is thinned until a beam-like representation consists. Second, based on the line thickness or the colored cross-section, the finite element analysis and the subdivision surface control grid estimation are covered. This beam representation serves for the 3D redesign.

\section{Beam Line Approximation Using Thinning and B-Spline Fitting}

The skeleton is approximated by applying a homotopic thinning method (Lee et al., 1994). The pixels of the image will be iteratively eroded until a chain having only onepixel in thickness is finally obtained. Based on that skeleton, a topological segmentation followed by B-spline fitting is applied. The following figure shows the different steps for the estimation of the beamline topology and its position.

\section{Cross-Section Extraction and FEM}

The determination of the cross-section covers two different approaches. First, if no cross-section is predefined, the line thickness of the skeleton is chosen as an approximation of the control grid similar to (Denk et al., 2020b). Second, if a predefined elliptical cross-section is chosen, the control grid is approximated using a bounding box. The following figure shows the two different strategies for the determination of the control grid for the subdivision surface model. 


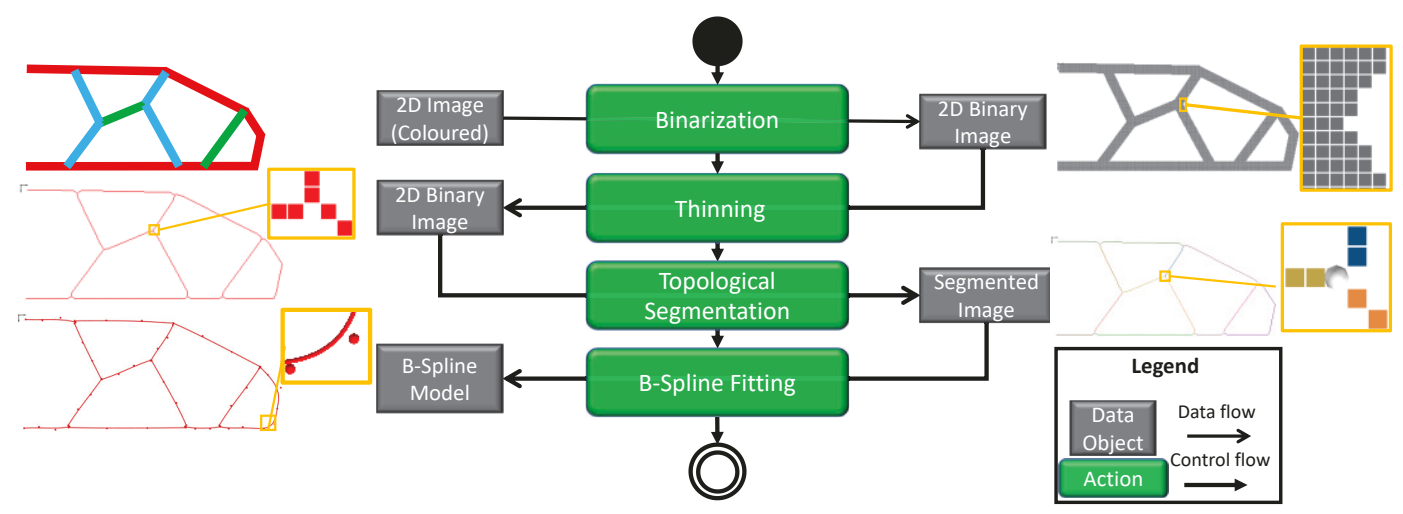

Figure 2: Beam Line Approximation

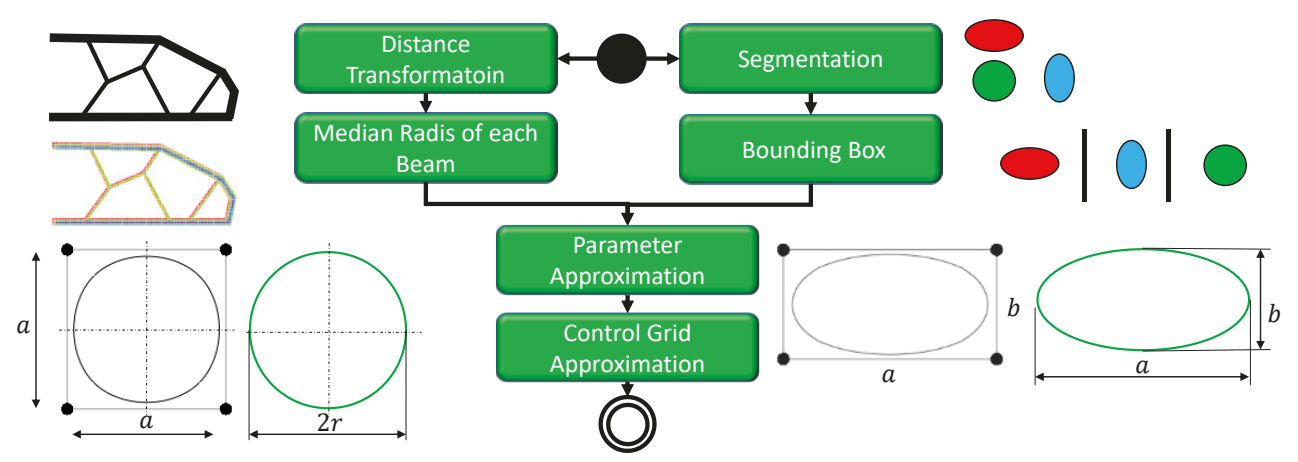

Figure 3: Radii determination

Based on these cross-section properties, a finite-element analysis can be directly applied to beam elements. The stiffness matrix for a 2D-truss element $\boldsymbol{k}_{e}$ can be computed by

$$
\boldsymbol{k}_{e}=\int_{\Omega} \boldsymbol{B}^{T} \boldsymbol{C B} d \Omega=A \int_{0}^{l} \boldsymbol{B}^{T} \boldsymbol{C B} d l=E \frac{A}{l}\left[\begin{array}{cc}
1 & -1 \\
-1 & 1
\end{array}\right],
$$

where $\boldsymbol{B}$ represents the strain-displacement transformation matrix and $\boldsymbol{C}$ the material matrix reducing to the modulus of elasticity $E, A$ the area of the cross-section and $l$ the length of the element (Rieg et al., 2012). For a 2D-beam element, the stiffness matrix can be computed with 


$$
\boldsymbol{k}_{e}=E \int_{A} y^{2} d A \int_{0}^{l}\left(\frac{\partial^{2}}{\partial x^{2}} \boldsymbol{N}\right)^{T}\left(\frac{\partial^{2}}{\partial x^{2}} \boldsymbol{N}\right) d x=E \frac{I}{l^{3}}\left[\begin{array}{cccc}
12 & 6 l & -12 & 6 l \\
6 l & 4 l^{2} & -6 l & 2 l^{2} \\
-12 & -6 l & 12 & -6 l \\
6 l & 2 l^{2} & -6 l & 4 l^{2}
\end{array}\right] \text {, }
$$

with the second moment of inertia I (Rieg et al., 2012). By assembling both stiffness matrices, a complete frame structure can be computed. The following figure shows the result of a colored sample using FEM. By changing the material properties for the red lines, the corresponding elements are stiffer so that the deformation is smaller.

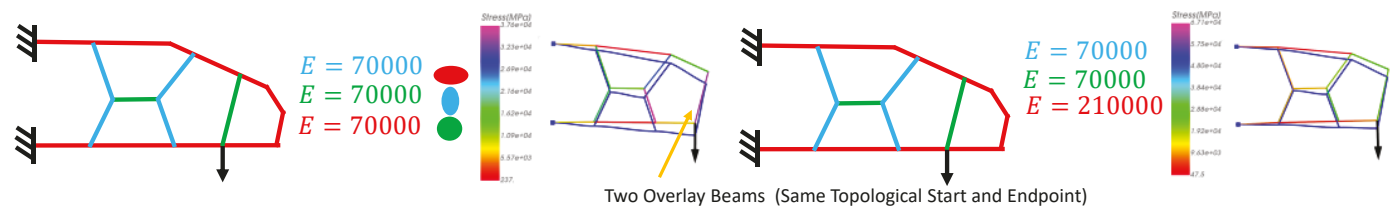

Figure 4: Sample of the topological segmented beam with the young module with $F=10000$

\section{Subdivision Surface Reconstruction}

The figure 5 shows the assembly of the skeleton and control points of the cross-section into a connected control grid mesh. For each beam along with the skeleton, several points are chosen where the control grid of the cross-section is applied. These control girds are connected to a beam control grid, which consists of a not-connected mesh for each beam. On the junctions, the corresponding control points of the connected beams are chosen to compute a convex hull. Such hull and the beams are combined using a Boolean operation on the mesh. This connected mesh can be subdivided for a smooth geometry.
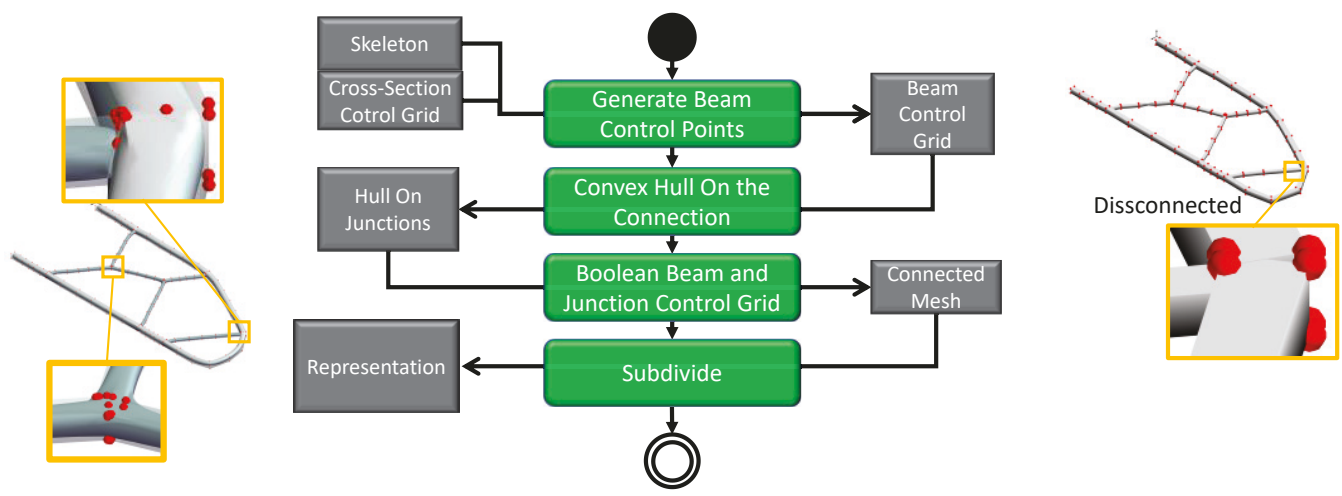

Figure 5: Subdivision Surface Reconstruction of the wireframe model 
The figure 6 shows the generation of the control grid of the cross-section by projecting them normal to the skeleton. Additionally, the generation of the Convex Hull is visualized for a junction.
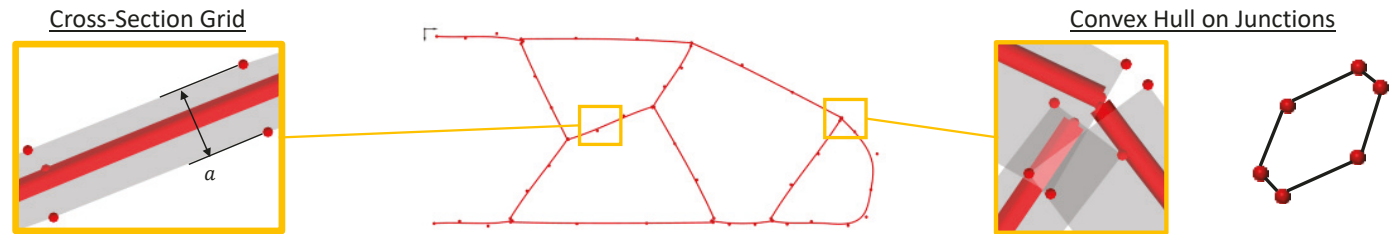

Figure 6: Control Grid Creation for Beams and Junctions

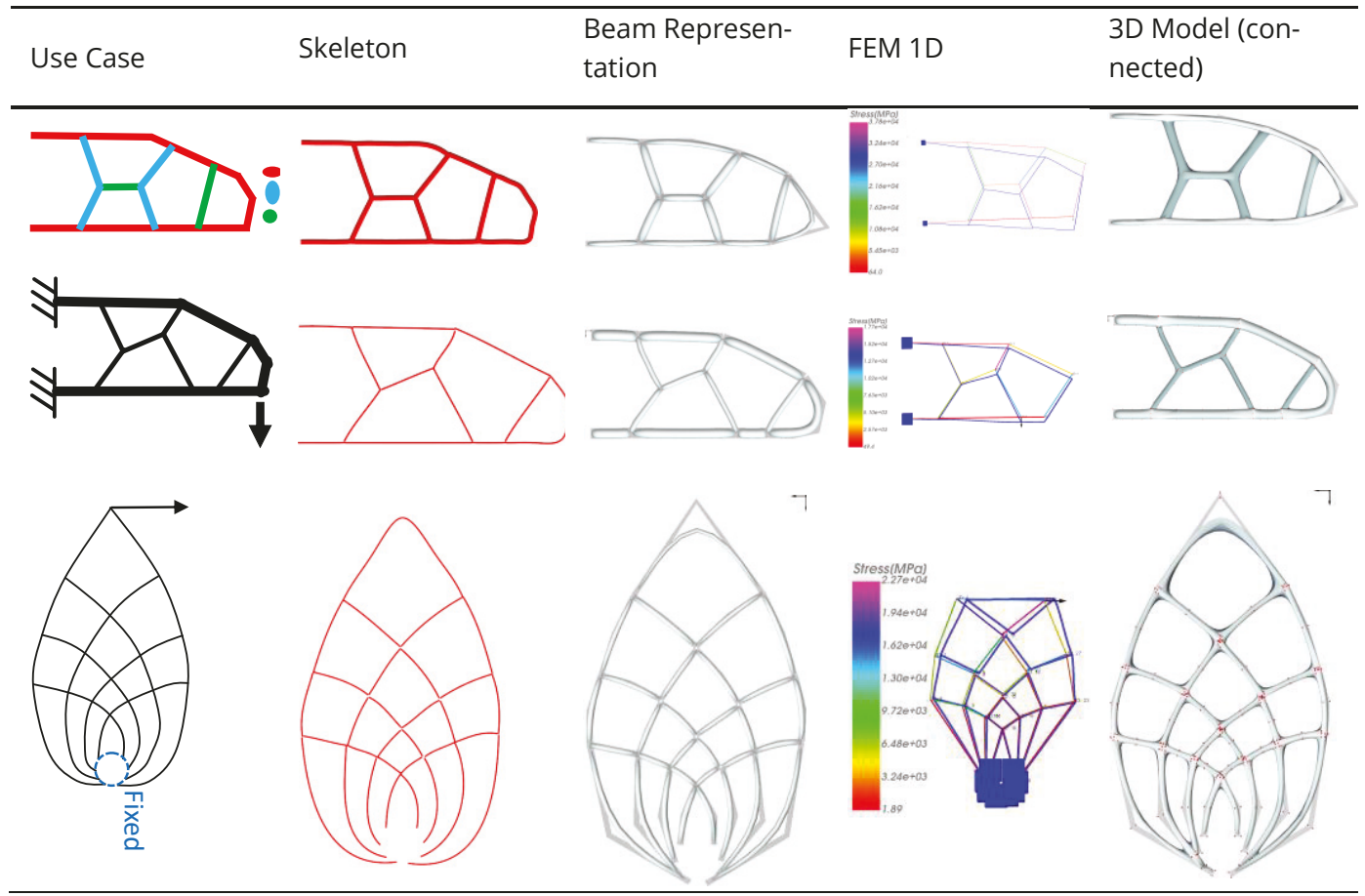

Table 1: Use-Cases of the wireframe reverse engineering 


\section{Experiment}

The experiment is separated into two parts. First, the sketch-based reconstruction on four samples is covered, showing the skeleton, the beam model, the Finite Element model, and the beams connected to the junctions. Table 1 shows the result using different colored cross-sections, different frame structures, and different line thicknesses.

In particular, the last results derived from Michell structures (M.C.E, 1904) show a robust approach covering complex shapes and a huge amount of junctions. Additionally, the figure 7 shows the example using different elliptical cross-sections depending on the orientation and the size of the designed section. The different sizes of the crosssection are smoothly connected to each other.

That proposed approach can also be applied to results provided by topology optimization. Table 2 covers different use-cases in topology optimization, such as simultaneous consideration of multiphysics, like a pareto-optimization of linear elastostatics heat transfer and fluid flow (Denk et al., 2020a).

Table 2: Reverse Engineering of 2D Results in Topology Optimization

Use Case Segmentation $\quad$ Skeleton $\quad$ Beam Grid $\quad \begin{aligned} & \text { Convex Hull con- } \\ & \text { nected }\end{aligned}$
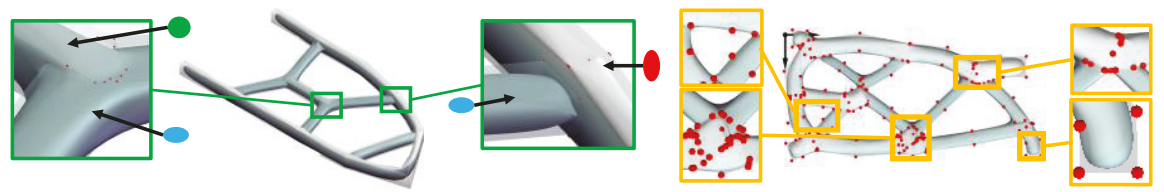

Figure 7: Sample showing the connection of different beams as junctions 


\section{Conclusion}

This article deals with the fully automated evaluation and 3D redesign of images covering beam-like shapes. The skeleton-like images can be provided by topology optimization, sketches, or drawing images. Based on the skeleton image, the beamlines are approximated using a thinning method. The cross-sections are determined by the Euclidian distance transformation or by predefined elliptical cross-section sketches. These cross-sections serve as a guideline for the control grid estimation for a subdivision surface grid. Based, on this beam representation, a finite element analysis is performed. For each topological segmented beam, one element is defined. Based on the color of the skeleton and predefined cross-section, different materials and cross-sections can be covered automatically. The subdivision surface beam grids need to be connected to each other. Therefore, this work uses a convex hull algorithm, which connects the control grid of each topological segmented beam on each junction. Afterward, this convex hull on the junction is united with the beam elements so that connectivity is ensured.

For wireframe sketches, optimized topology results, or Michell structures shown in (M.C.E, 1904), this approach leads to a reasonable result. The topology and each branch are covered but it can sometimes lack in covering the size of cross-sections. Due to the convex hull on each junction, quite a number of polygons are generated, which increases the number of points particular in that region. For further processing, the points on these junctions should be reduced. To cover a better approximation of the beams, a more variable cross-section can be applied along each beam.

\section{Literature}

Adam, G.A.0., Zimmer, D., 2015. On design for additive manufacturing: evaluating geometrical limitations. Rapid Prototyp. J. 21, 662-670. https://doi.org/10.1108/RPJ-06-2013-0060

Agathos, A., Pratikakis, I., Perantonis, S., Sapidis, N., Azariadis, P., 2007. 3D Mesh Segmentation Methodologies for CAD applications. Comput.-Aided Des. Appl. 4, 827-841. https://doi.org/10.1080/16864360.2007.10738515

Bandara, K., Rüberg, T., Cirak, F., 2016. Shape optimisation with multiresolution subdivision surfaces and immersed finite elements. Comput. Methods Appl. Mech. Eng. 300, 510-539. https://doi.org/10.1016/j.cma.2015.11.015

Ben Makhlouf, A., Louhichi, B., Mahjoub, M.A., Deneux, D., 2019. Reconstruction of a CAD model from the deformed mesh using B-spline surfaces. Int. J. Comput. Integr. Manuf. 32, 669-681. https://doi.org/10.1080/0951192X.2019.1599442

Bénière, R., Subsol, G., Gesquière, G., Le Breton, F., Puech, W., 2013. A comprehensive process of reverse engineering from 3D meshes to CAD models. Comput.-Aided Des. 45, 1382-1393. https://doi.org/10.1016/j.cad.2013.06.004 
Berger, M., Tagliasacchi, A., Seversky, L.M., Alliez, P., Guennebaud, G., Levine, J.A., Sharf, A., Silva, C.T., 2017. A Survey of Surface Reconstruction from Point Clouds: A Survey of Surface Reconstruction from Point Clouds. Comput. Graph. Forum 36, 301-329. https://doi.org/10.1111/cgf.12802

Blum, H., 1967. A Transformation for Extracting New Descriptors of Shape. M.I.T. Press.

Bremicker, M., Chirehdast, M., Kikuchi, N., Papalambros, P.y., 1991. Integrated Topology and Shape Optimization in Structural Design*. Mech. Struct. Mach. 19, 551-587. https://doi.org/10.1080/08905459108905156

Changizi, N., Warn, G.P., 2020. Topology optimization of structural systems based on a nonlinear beam finite element model. Struct. Multidiscip. Optim. https://doi.org/10.1007/s00158-020-02636-x

Denk, M., Paetzold, K., Rother, K., 2019. Feature line detection of noisy triangulated CSGbased objects using deep learning, in: Proceedings of the 30th Symposium Design for X (DFX 2019), DfX. Presented at the DfX Symposium 2019, The Design Society, Jesteburg, Germany, pp. 239-250. https://doi.org/10.35199/dfx2019.21

Denk, M., Rother, K., Paetzold, K., 2020a. Multi-Objective Topology Optimization of Heat Conduction and Linear Elastostatic using Weighted Global Criteria Method, in: Proceedings of the 31st Symposium Design for X (DFX2020), DFX. Presented at the DfX Symposium 2020, The Design Society, Bamberg, pp. 91-100. https://doi.org/10.35199/dfx2020.10

Denk, M., Rother, K., Paetzold, K., 2020b. Fully Automated Subdivision Surface Parametrization for Topology Optimized Structures and Frame Structures using Euclidean Distance Transformation and Homotopic Thinning, in: Proceedings of the Munich Symposium on Lightweight Design 2020. Springer Nature, Munich, Germany. https://doi.org/10.1007/978-3-66263143-0

Ensici, A., Badke-Schaub, P., 2011. Information behavior in multidisciplinary design teams, in: Proceedings of the 18th International Conference on Engineering Design (ICED 11). Presented at the Proceedings of the 18th International Conference on Engineering Design (ICED 11), Denmark, pp. 414-423.

Feng, C., Jalba, A.C., Telea, A.C., 2015. Part-Based Segmentation by Skeleton Cut Space Analysis, in: Benediktsson, J.A., Chanussot, J., Najman, L., Talbot, H. (Eds.), Mathematical Morphology and Its Applications to Signal and Image Processing, Lecture Notes in Computer Science. Springer International Publishing, Cham, pp. 607-618. https://doi.org/10.1007/978-3319-18720-4_51

Gao, J., Luo, Z., Xiao, M., Gao, L., Li, P., 2020. A NURBS-based Multi-Material Interpolation (N-MMI) for isogeometric topology optimization of structures. Appl. Math. Model. 81, 818-843. https://doi.org/10.1016/j.apm.2020.01.006

Guo, Y., Bennamoun, M., Sohel, F., Lu, M., Wan, J., Kwok, N.M., 2016. A Comprehensive Performance Evaluation of 3D Local Feature Descriptors. Int. J. Comput. Vis. 116, 66-89. https://doi.org/10.1007/s11263-015-0824-y

Lee, T.C., Kashyap, R.L., Chu, C.N., 1994. Building Skeleton Models via 3-D Medial Surface Axis Thinning Algorithms. CVGIP Graph. Models Image Process. 56, 462-478. https://doi.org/10.1006/cgip.1994.1042

Louhichi, B., Abenhaim, G.N., Tahan, A.S., 2015. CAD/CAE integration: updating the CAD model after a FEM analysis. Int. J. Adv. Manuf. Technol. 76, 391-400. https://doi.org/10.1007/s00170-014-6248-y

Mayer, J., Wartzack, S., 2020. Ermittlung eines Skelettierungsverfahrens zur Konvertierung von Topologieoptimierungsergebnissen, in: Proceedings of the 31st Symposium Design for X (DFX2020). Presented at the Symposium Design for X 2020, Bamberg, pp. 111-120. https://doi.org/10.35199/dfx2020.12 
M.C.E, A.G.M.M., 1904. LVIII. The limits of economy of material in frame-structures. Lond. Edinb. Dublin Philos. Mag. J. Sci. 8, 589-597. https://doi.org/10.1080/14786440409463229

Nana, A., Cuillière, J.-C., Francois, V., 2017a. Automatic reconstruction of beam structures from 3D topology optimization results. Comput. Struct. 189, 62-82. https://doi.org/10.1016/j.compstruc.2017.04.018

Nana, A., Cuillière, J.-C., Francois, V., 2017b. Automatic reconstruction of beam structures from 3D topology optimization results. Comput. Struct. 189, 62-82. https://doi.org/10.1016/j.compstruc.2017.04.018

Reniers, D., Telea, A., 2008. Part-type Segmentation of Articulated Voxel-Shapes using the Junction Rule. Comput. Graph. Forum 27, 1845-1852. https://doi.org/10.1111/j.1467-8659.2008.01331.x

Rieg, F., Hackenschmidt, R., Alber-Laukant, B., 2012. Finite Elemente Analyse für Ingenieure, 4., überarbeitete Edition. ed. Carl Hanser Verlag GmbH \& Co. KG, München.

Song, S., 2004. Shared Understanding, Sketching, and Information Seeking and Sharing Behavior in the New Product Design Process. University of California, Berkeley.

Stangl, T., Wartzack, S., 2015. Feature based interpretation and reconstruction of structural topology optimization results, in: Weber, M., C.,. Husung, S.;. Cascini, G.;.: Cantamessa, M.;. Marjanovic, D.,. Bordegoni (Ed.), Proceedings of the 20th International Conference on Engineering Design (ICED15). Design Society, p. Vol. 6, 235-245.

Vidal, V., Wolf, C., Dupont, F., 2014. Mechanical Mesh Segmentation and Global 3D Shape Extraction.

Wu, B., Xu, K., Zhou, Y., Xiong, Y., Huang, H., 2016. Skeleton-guided 3D shape distance field metamorphosis. Graph. Models, SIX: CVM 2016 selected Papers 85, 37-45. https://doi.org/10.1016/j.gmod.2016.03.003

Xu, K., Chen, K., Fu, H., Sun, W.-L., Hu, S.-M., 2013. Sketch2Scene: sketch-based co-retrieval and co-placement of 3D models. ACM Trans. Graph. 32, 123:1-123:15. https://doi.org/10.1145/2461912.2461968

Yin, G., Xiao, X., Cirak, F., 2020. Topologically robust CAD model generation for structural optimisation. Comput. Methods Appl. Mech. Eng. 369, 113102. https://doi.org/10.1016/j.cma.2020.113102

Zhao, X., Gregor, R., Mavridis, P., Schreck, T., 2017. Sketch-based 3D Object Retrieval with Skeleton Line Views - Initial Results and Research Problems. The Eurographics Association. https://doi.org/10.2312/3dor.20171052

\section{Contact}

Martin Denk, M. Sc.

Prof. Dr.-Ing. Kristin Paetzold

Bundeswehr University Munich

Institute for Technical Product Development

Werner-Heisenberg-Weg 39

85577 Neubiberg

unibw.de/itpe
Prof. Dr.-Ing. Klemens Rother

Munich University of Applied Science

Institute for Material and Building Research

Lothstraße 64

80335 München

sites.hm.edu/imb/ 